\title{
A SINGLE-CRYSTAL SILICON RESONATOR FOR AM DEMODULATION WITH ADDED SECOND-HARMONIC MODULATION
}

\author{
Mary E. Galanko ${ }^{*}$, Yi-Chung Lin ${ }^{l}$, Tamal Mukherjee ${ }^{l}$, and Gary K. Fedder ${ }^{1,2}$ \\ ${ }^{1}$ Department of Electrical and Computer Engineering and ${ }^{2}$ The Robotics Institute \\ Carnegie Mellon University, Pittsburgh, PA
}

\begin{abstract}
We report a single-crystal silicon MEMS resonator that functions as a demodulator in a near-zero-power wake-up receiver. The receiver detects an AM signal at a certain carrier frequency $f_{\mathrm{c}}$ and primary modulation frequency $f_{\mathrm{m}}$; the demodulator resonates and filters at $f_{\mathrm{m}}$ and leverages the quadratic dependence of electrostatic force upon input voltage to convert from highfrequency to baseband. Two key advantages are passive operation and very high out-of-band rejection. In order to increase the sensitivity of the demodulator, we propose the use of additional second-harmonic modulation to increase the response amplitude for a given AM input power. This work considers both the resulting parametric force, which is negligible, and additional direct force, a significant component. This device is the first to our knowledge to demonstrate multi-harmonic mixing and investigate parametric amplification in response to an amplitude-modulated (AM) signal with added modulation at a second-harmonic pumping frequency.
\end{abstract}

\section{INTRODUCTION}

A "wake-up" receiver (WuR) is designed to detect the presence of a particular signal while consuming very little power. When a signal is present, the $\mathrm{WuR}$ produce a voltage change that triggers higher-power circuitry to process the signal, so that the radio is continuously alert to the signal yet does not consume this high processing power when no signal is present. The bulk Si MEMS resonator in this work serves as a near-zero-power demodulator for use in a WuR. This device builds upon past work and designs [1] in a different technology while exploring the nonlinear response of these devices to multi-frequency modulation. This modulation scheme has not been considered in this application but carries advantages in demodulator sensitivity.

\section{Motivation}

To maximize battery life and hence maximize the advantage of a WuR, it should operate at as little power as possible. The active blocks of an AM receiver generally include a front-end filter, a demodulator, and baseband signal processing circuitry. One approach [2] to minimizing power is to implement the front-end and demodulator at near-zero power using passive MEMS devices, so that the only significant power consumption is in the final baseband stages, which may consume only tens of $\mathrm{nW}$.

The front-end is a lithium niobate (LN) piezoelectric resonator [3], which operates around 40-400 MHz, filtering and increasing the voltage of AM signals with a particular carrier frequency in that range. The demodulator is a capacitive MEMS resonator, operating around $50-200 \mathrm{kHz}$. As recently demonstrated in a CMOS-MEMS implementation [1], the demodulator leverages the quadratic dependence of capacitive electrostatic force upon drive voltage to generate forces at various frequencies, including the resonance $f_{\mathrm{r}}$. The system will only respond significantly and produce a "wakeup" signal when the demodulator is forced at $f_{\mathrm{r}}$ via to the modulation frequency $f_{\mathrm{m}}$ of the input signal. This property affords the MEMS demodulator a major advantage in false alarm rate compared with a CMOS envelope detector, where sharply filtering around $f_{\mathrm{m}}$ would consume too much additional power.
In this complex, multi-technology system, system-level optimization is critical to achieve performance goals. Given design limits of each device, impedance matching between all three stages is the primary factor in performance [2]. This system context impacts the MEMS demodulator technology and design choices.

\section{Background}

Several past works [4-6] have proposed the use of MEMS devices as passive mixers. Koskenvuori et al. [7] implemented a capacitive MEMS resonant mixer that used the same quadratic mechanism to convert an AM signal to baseband, but noted the problem of low sensitivity and conversion efficiency. This problem is always a concern in designs that spread the input signal power in frequency, but is addressed in [1] by system-level optimization that identifies a matched design for sufficient sensitivity.

Few works have explored multi-frequency forcing and mixing. Koskenvuori and Tittonen proposed a modified resonant mixer design that improves sensitivity via parametric amplification [8]. Thompson and Horsley [9] have shown that parametric amplification can increase the sensitivity of a magnetometer. However, both of these works required a local oscillator. We investigate the possible advantages of multi-frequency mixing in a demodulator specifically for a near-zero-power RF WuR. Because the power budget precludes the use of a local oscillator, this work uniquely considers force variations created by changing the modulation of a low-power AM input signal. This condition greatly limits the use of parametric amplification, but brings advantages in direct force.

The "Epi-Seal" process [10], in $60 \mu \mathrm{m}$ bulk Si, is used partly because it offers larger capacitances and better impedance matching according to the findings in [2] relative to CMOS-MEMS. A CMOS-MEMS demodulator offers direct integration with the baseband circuit, but less capacitance per unit area is possible than in a very thick bulk Si process. For a given gap size, higher capacitance per unit area also maximizes parametric force, providing a better platform to test feasibility of parametric forcing.

\section{MULTI-SIDEBAND FORCING}

Figure 1 shows an equivalent circuit for the demodulator. The electrostatic force acts upon a basic mechanical resonator to produce an AC output current $i_{\text {mot }}$ proportional to velocity and polarization $V_{\mathrm{P}}$. Assuming no loading by the readout circuit, and neglecting

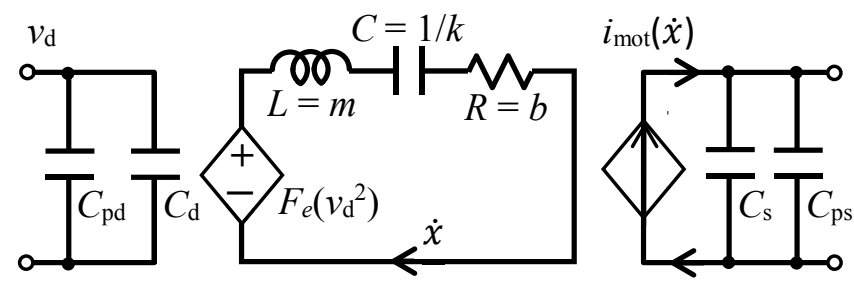

Figure 1: Equivalent circuit of the demodulator, a mass-springdamper with force $F_{e} \propto v_{d}{ }^{2}$. Input and output loads include device capacitances $C_{d}, C_{s}$ and parasitic capacitances $C_{p d}, C_{p s}$. Hilton Head Island, South Carolina, June 3-7, 2018 
mechanical and electrostatic nonlinearities due to small displacement, the demodulator is governed by the canonical equation of motion (EoM) in displacement $x$ :

$$
m \ddot{x}+b \dot{x}+k_{e f f} x=F_{e}
$$

where $m$ is the effective mass of the device; $b$ is the damping coefficient; and $k_{\text {eff }}=k_{0}+k_{\mathrm{e}}$ is the effective spring constant after electrostatic softening $k_{\mathrm{e}}<0$ of the mechanical spring $k_{0}$.

The demodulator is sensitive to arbitrary input waveform shapes and carrier frequencies. It mixes the voltage $V_{\mathrm{d}}$ across its drive capacitance $C_{\mathrm{d}}$ according to

$$
F_{e}=\frac{1}{2} V_{d}^{2} \frac{\partial C_{d}}{\partial x}
$$

Plugging an AM waveform with amplitude $V_{\text {in }}$ into (2) yields

$$
\begin{aligned}
F_{e} & =\frac{1}{2} \frac{\partial C_{d}}{\partial x}\left[V_{\text {in }} \cos \left(\omega_{c} t\right) \cdot 0.5\left(1+\cos \left(\omega_{m} t\right)\right)\right]^{2} \\
& =\frac{V_{\text {in }}{ }^{2}}{8}\left(\frac{1+\cos \left(2 \omega_{c} t\right)}{2}\right)\left(\frac{1+\cos \left(2 \omega_{m} t\right)}{2}+2 \cos \left(\omega_{m} t\right)+1\right)
\end{aligned}
$$

The largest low-frequency force component is at $\omega_{\mathrm{m}}$. If $\omega_{\mathrm{m}}=\omega_{\mathrm{r}}$, this force drives the demodulator into resonance. There is also a smaller force component at $2 \omega_{\mathrm{m}}$.

From this force equation, we aim to quantify the direct and parametric forces possible during $\mathrm{AM}$ demodulation and measure their effects. Neglecting out-of-band force terms, the EoM may be rewritten successively from (1), (3) to reach the final form

$m \ddot{x}+b \dot{x}+k_{e f f} x=F_{1} \cos \left(\omega_{m} t\right)+F_{2} \cos \left(2 \omega_{m} t\right)$

$m \ddot{x}+b \dot{x}+\left(k_{e f f}+\Delta k \sin \left(2 \omega_{m} t\right)\right) x=F_{1} \cos \left(\omega_{m} t+\varphi\right)$

where

$F_{1}=\frac{V_{i n}{ }^{2}}{8} \frac{\partial C_{d}}{\partial x}, F_{2}=\frac{V_{i n}^{2}}{16} \frac{\partial C_{d}}{\partial x}, \Delta k=-\frac{\partial^{2} C_{d}}{\partial x^{2}} \frac{V_{i n}{ }^{2}}{16}$

and $\varphi=\pi / 2$ using the input waveform in (5) where the direct and parametric forces are in phase. Past work $[9,11]$ has shown that in systems of this EoM form, the parametric forcing term results in an output current amplification factor

$$
\begin{gathered}
G=\left[\left(\frac{\cos \varphi^{\prime}}{1+Q \Delta k / 2 k_{0}}\right)^{2}+\left(\frac{\sin \varphi^{\prime}}{1-Q \Delta k / 2 k_{0}}\right)^{2}\right]^{1 / 2} \\
\left.G\right|_{\varphi=\pi / 2}=\left(1-Q \Delta k / 2 k_{0}\right)^{-1 / 2}
\end{gathered}
$$

For values achievable in the Epi-Seal demodulator $(Q=10000$, $\left.\Delta k=10^{-4} \mathrm{~N} / \mathrm{m}, k=130 \mathrm{~N} / \mathrm{m}, V_{\text {in }}=30 \mathrm{mV}\right)$, this amplification is not significant $(<1 \%)$.

Adding AM sidebands at $2 \omega \mathrm{m}$ with generic amplitude $B$ and phase $\varphi$ relative to the modulation at $\omega_{\mathrm{m}}$, the input force (3) becomes

$$
\begin{aligned}
F_{e}= & \frac{1}{2} \frac{\partial C_{d}}{\partial x}\left[V_{\text {in }} \cos \left(\omega_{c} t\right)\right. \\
& \left.\cdot 0.5\left(1+\cos \left(\omega_{m} t\right)+B \cos \left(2 \omega_{m} t+\varphi\right)\right)\right]^{2}
\end{aligned}
$$

Expanding and isolating force terms at $\omega_{\mathrm{m}}$ and $2 \omega \mathrm{m}$ yields
$F_{e}=\frac{V_{i n}{ }^{2}}{8}\left(\frac{1+\cos \left(2 \omega_{c} t\right)}{2}\right)\left[\frac{1}{2} \cos \left(2 \omega_{m} t\right)\right.$

$\left.+2 B \cos \left(2 \omega_{m} t+\varphi\right)+2 \cos \left(\omega_{m} t\right)+B \cos \left(\omega_{m} t+\varphi\right)\right]$

These additional terms change $\Delta k$ only incrementally, resulting in only minimal change in the parametric amplification gain $G(10)$. Conversely, the direct force increases significantly with $B$ :

$$
\begin{gathered}
\Delta k=-\frac{\partial^{2} C_{d}}{\partial x^{2}} \frac{V_{\text {in }}{ }^{2}}{16}\left[2 B \cos (\varphi)+\frac{1}{4}+4 B^{2}\right]^{1 / 2} \\
\left.F_{e}\right|_{\omega=\omega_{m}}=\frac{V_{\text {in }}{ }^{2}}{16} \frac{\partial C_{d}}{\partial x}\left[B^{2}+4 B \cos \varphi+4\right]^{1 / 2}
\end{gathered}
$$

The addition of $2 \omega_{\mathrm{m}}$ sidebands also changes the ratio of force at $\omega_{\mathrm{m}}$ to total normalized input signal power $P_{0}$ :

$$
\begin{gathered}
P_{0}=P_{\text {in }} \cdot Z_{\text {in }}=V_{d}^{2}(t)=\frac{1}{2}\left(\left(\frac{V_{\text {in }}}{2}\right)^{2}+2\left(\frac{V_{\text {in }}}{2}\right)^{2}+2\left(\frac{B \cdot V_{\text {in }}}{2}\right)^{2}\right) \\
=\frac{V_{\text {in }}^{2}}{16}\left(B^{2}+3\right) \\
\frac{\left.F_{e}\right|_{\omega=\omega_{m}}}{P_{\text {in }, 0} \cdot Z_{\text {in }}}=\frac{\partial C_{d}}{\partial x} \frac{\left[B^{2}+4 B \cos \varphi+4\right]^{1 / 2}}{B^{2}+3}
\end{gathered}
$$

This relationship is not monotonic but results in greater $F_{\mathrm{e}}$ for fixed $P_{0}$ for certain sideband amplitudes $B$. The device and tests in this work validate these models.

\section{DEMODULATOR DESIGN}

The Epi-Seal process fabricates devices in a $60 \mu \mathrm{m}$ silicon-oninsulator (SOI) device layer using a Bosch silicon etch process with on-chip epitaxial vacuum encapsulation $[10,12]$. The process is well-understood and reliable for certain applications, but for lowfrequency capacitive resonators, it presents yield risks and imposes important design constraints. The maximum silicon etch aspect ratio restricts the minimum layout gap size to $0.7 \mu \mathrm{m}$, and the cap deposition process limits the maximum width of a continuous released area to about $450 \mu \mathrm{m}$ [12]. Furthermore, past work in this process has shown that low-spring constant, high-capacitance EpiSeal resonators at around $100 \mathrm{kHz}$ are prone to post-release stiction.

Figure 2 shows a layout view of the resulting demodulator design. It consists of a proof mass with capacitive rotor comb fingers, anchored through in-plane springs. The rotor fingers

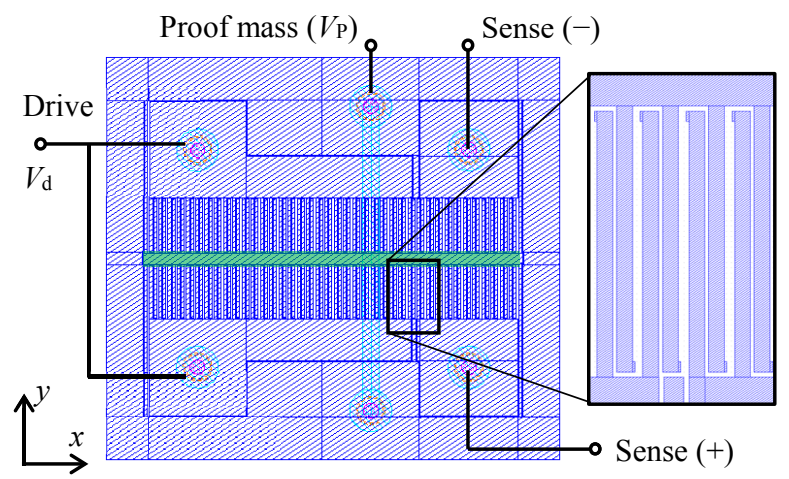

Figure 2: Epi-Seal demodulator layout diagram. Green denotes the proof mass, with fixed-fixed spring beams on either end. The inset shows the capacitive comb finger geometry. The device moves in $x$. 
interface with fixed stator comb fingers for drive and sense transduction. The device moves perpendicular to the fingers so that resonant motion varies the parallel-plate gap size. $V_{\mathrm{P}}$ is applied across the sense comb fingers to produce an output motional current $i_{\text {mot }}$ proportional to this displacement:

$$
i_{m o t}(t)=V_{P} \frac{\partial C_{x}}{\partial x} \frac{\partial x}{\partial t} \approx V_{P} \frac{\partial C_{x}}{\partial x} \frac{F_{e} Q}{k_{e f f}}
$$

To prevent substrate stiction, the proof mass area is minimized, and the springs are fixed-fixed beams designed to maximize out-ofplane stiffness. The risk of stiction between the rotor and stator comb fingers depends mostly on the device capacitance (i.e., comb finger area and geometry) and frequency. For this application, the demodulator resonant frequency was fixed at around $50 \mathrm{kHz}$, and among designs with various total capacitance, we use the highestcapacitance device that is found to be fabricated without in-plane stiction. The device contains a total of $N_{\mathrm{d}}=58$ drive and $N_{\mathrm{s}}=22$ sense comb fingers. These and other design values are summarized in Table 1 .

Table 1: Quantitative details of the demodulator design used.

\begin{tabular}{|l|l|c|c|}
\hline Variable & \multicolumn{1}{|c|}{ Description } & Value & Unit \\
\hline$m_{\mathrm{eff}}$ & Effective mass & $1.77 \cdot 10^{-9}$ & $\mathrm{~kg}$ \\
\hline$k_{\mathrm{eff}}$ & Effective spring & 132 & $\mathrm{~N} / \mathrm{m}$ \\
\hline$\Delta k$ & Parametric $k$ variation & $8.29 \cdot 10^{-5}$ & $\mathrm{~N} / \mathrm{m}$ \\
\hline$C_{1}$ & Comb pair capacitance & 16.7 & $\mathrm{fF}$ \\
\hline$N_{\mathrm{d}}$ & \# drive comb fingers & 58 & - \\
\hline$N_{\mathrm{s}}$ & \# sense comb fingers & 22 & - \\
\hline
\end{tabular}

The comb finger capacitance $C_{1}$ is adjusted to measurements by modifying the gap size in calculations to correct for bloat. A bloat of about $400 \mathrm{~nm}$ per side from the layout gap dimension of $0.7 \mu \mathrm{m}$ gives a comb finger width of about $2.2 \mu \mathrm{m}$, as validated in Figure 3, and total device capacitance of about $2.1 \mathrm{pF}$, which agrees reasonably with measurements, as detailed in the following section.

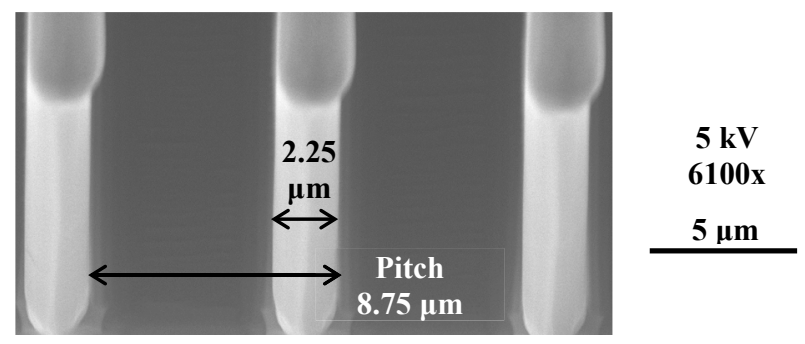

Figure 3: SEM image of an axial view of demodulator comb fingers (stators only), showing the finger width and pitch, which validate the effective parallel plate gap size used in analysis.

\section{RESULTS}

Basic frequency response measurements are performed first to characterize the resonator, followed by tests to validate models (14)(16) for $2 \omega_{\mathrm{m}}$ demodulation. Figure 4 shows a frequency response for direct harmonic forcing, where a polarization $V_{\mathrm{P}}$ is applied across both drive and sense comb fingers, so that the output current is a response to a low-frequency $\mathrm{AC}$ voltage and force at $f_{\mathrm{m}}$. Drive voltages $<1 \mathrm{mV}$ are still in the linear regime. In the nonlinear regime, there is a hardening nonlinearity contributed by the fixedfixed beam springs and an electrostatic softening nonlinearity from the parallel-plate comb fingers, but the mechanical hardening

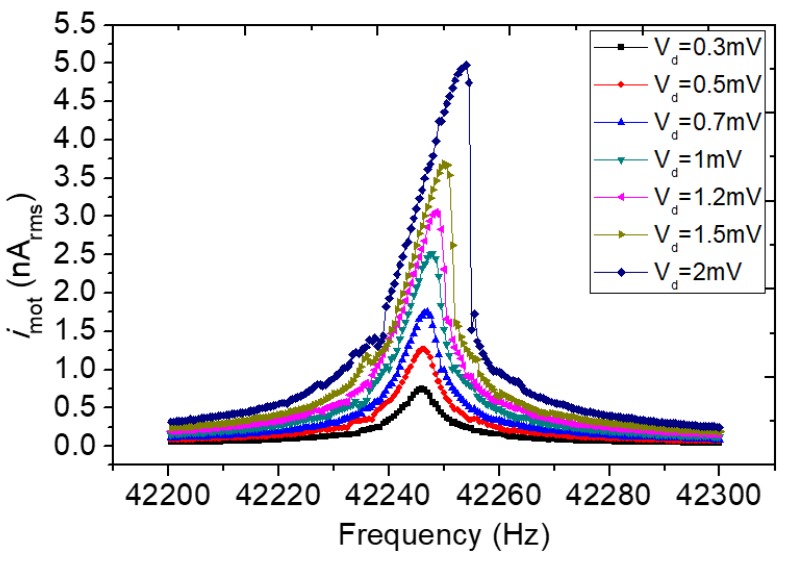

Figure 4: Frequency response of the resonant demodulator to direct harmonic drive for $V_{P}=2.5 \mathrm{~V}$ and various $A C$ drive amplitudes $V_{d}$.

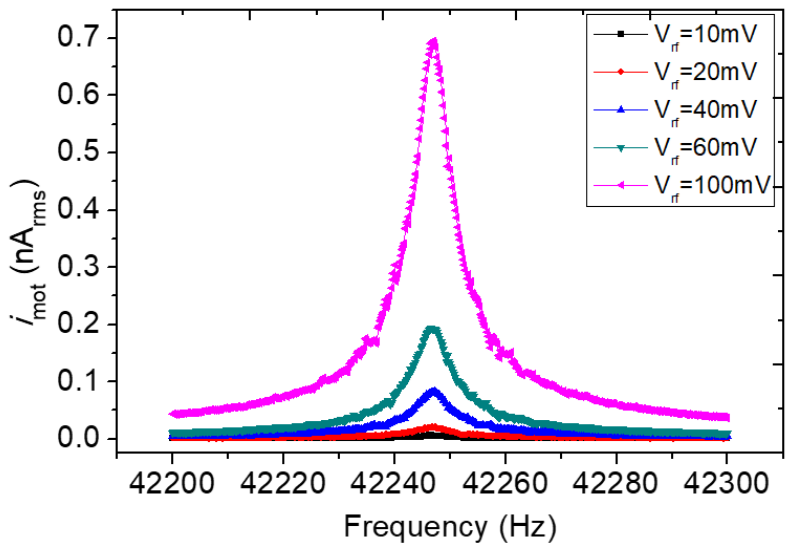

Figure 5: Demodulation of AM input signal with amplitude $V_{\text {in }}=V_{r f}$, in response to a sweep of the modulation frequency around resonance. The sense polarization is $V_{P}=2.5 \mathrm{~V}$; the drive comb fingers have no DC bias. The response scales quadratically with $V_{i n}$.

nonlinearity is greater. Focusing on linear $k_{\text {eff }}$ to simplify analysis and focus on the small signals relevant to the WuR, these linear responses agree with models within about 15\%. Figure 5 shows pure AM forcing results, with no second-harmonic modulation. These measurements also reasonably agree with calculations, within about $15 \%$ for low amplitudes in the linear regime.

Next, sidebands at $2 \omega \mathrm{m}$ were added to the original AM input signal, as depicted in Figure 6. As the relative amplitude $B$ and phase $\varphi$ vary, the response scales in agreement with the expected amplification relation in (16). For fixed $B=1$, Figure 7(a) shows response scaling for various $\varphi$; these values agree with expected scaling (16) within about $17 \%$. Phase $\varphi=0$ yields a maximal

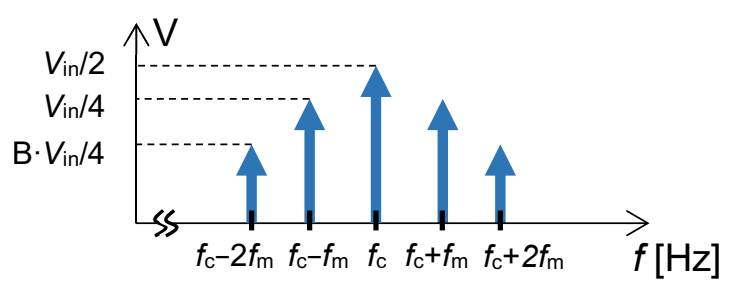

Figure 6: Frequency domain representation of the AM signal with second-harmonic modulation of relative amplitude $B$. 


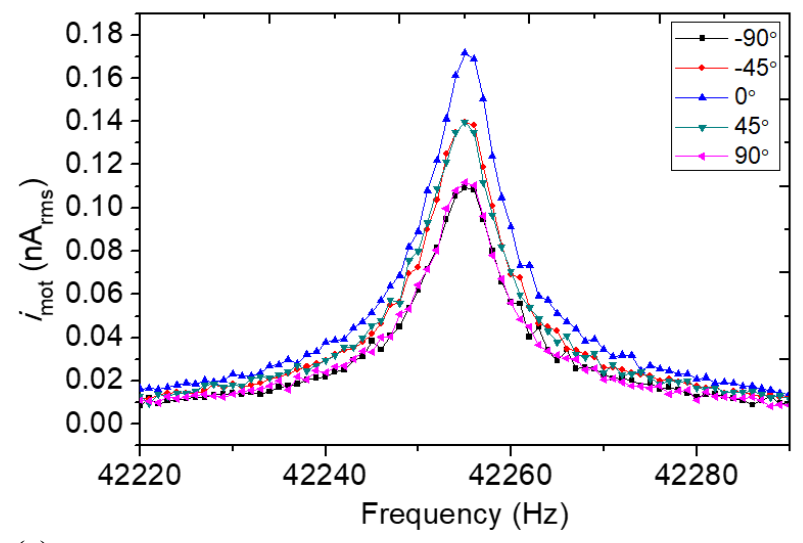

(a)

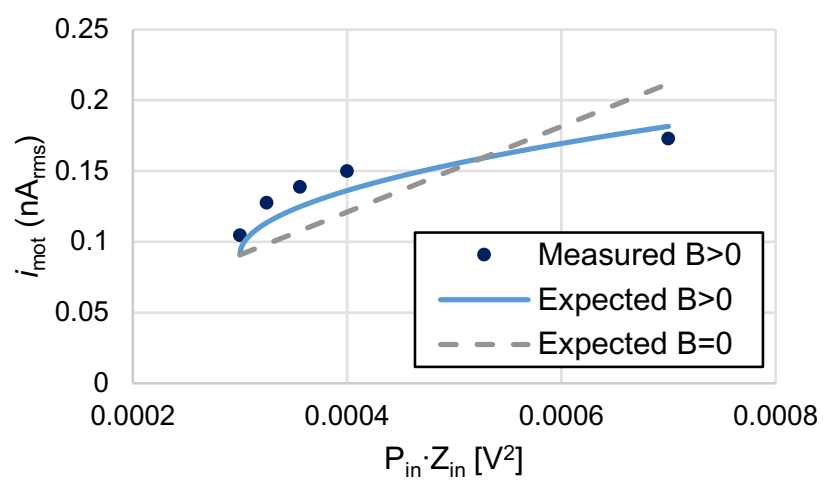

(b)

Figure 7: (a) Response with $V_{\text {in }}=40 \mathrm{mV}, B=2$, and various phases $\varphi$ of the $2 \omega_{m}$ modulation relative to the $\omega_{m}$ modulation. (b) Response to similar signals with $V_{\text {in }}=40 \mathrm{mV}, \varphi=0$, and various relative $2 \omega_{m}$ modulation amplitudes $B$. For $B>0$, power varies only by varying $B ; B=0$ data represent pure AM signals at the same power.

response increase as expected. Figure 7(b) shows scaling for various $B$ and $P_{0}$, also in agreement with (14-16), with an average error of about $11 \%$. For $B \approx 0.5$, there is an increase in response normalized to input power. Intuitively, this result and (16) show that adding sidebands at $2 \omega_{\mathrm{m}}$ marginally increases the convolution product at baseband and hence may increase the efficiency of input signal power to resonant force, but for large $B$, too much input signal power goes into other force components, diminishing efficiency.

\section{CONCLUSIONS}

This work presents a single-crystal vacuum-encapsulated bulk silicon resonant demodulator for a wake-up receiver. The device resonates in response to AM signals with any carrier frequency and any modulation envelope shape such that the quadratic force vs. $V_{\text {in }}$ relationship yields a force at the resonance $\omega_{\mathrm{m}}$. For certain device designs and input signals, modulation at both $\omega_{\mathrm{m}}$ and $2 \omega_{\mathrm{m}}$ may increase the power of the post-mixing force component at $\omega_{\mathrm{m}}$, for a given input signal power, and hence increase device performance. A $2 \omega_{\mathrm{m}}$ modulation also increases the force at frequency $2 \omega_{\mathrm{m}}$, but significant parametric amplification is difficult to achieve solely from this low input force rather than a local oscillator.

In summary, using an input waveform of this form incrementally increases demodulator sensitivity for certain $B$, but additional design techniques are needed to achieve orders-ofmagnitude improvement in sensitivity. However, the demonstration of multi-harmonic direct forcing in this work shows that a MEMS resonant $\mathrm{WuR}$ may be tuned to complex multi-frequency signals, while offering the same power and out-of-band rejection advantages that this architecture offers for simple AM input signals.

\section{ACKNOWLEDGMENTS}

This work was supported by the Defense Advanced Research Projects Agency (DARPA) under contract No. HR0011-15-C-0137. Any opinions, findings, and conclusions or recommendations expressed in this publication are those of the authors and do not necessarily reflect the views of DARPA.

Demodulator fabrication was performed by the Micro Structures \& Sensors Lab at Stanford University. The authors thank the principal investigator, Prof. T.W. Kenny, and thank L.C. Ortiz and D. Shin for valuable fabrication and layout support.

\section{REFERENCES}

[1] M.E. Galanko, A. Kochhar, G. Piazza, T. Mukherjee, and G.K. Fedder, "CMOS-MEMS resonant demodulator for near-zeropower RF wake-up receiver," in TRANSDUCERS 2017, Kaohsiung, Taiwan, 2017, pp. 86-89.

[2] L. Colombo, M. Galanko, H. Abdelsalam, G. Vidal-Álvarez, T. Mukherjee, J. Paramesh, J. Weldon, G.K. Fedder, and G. Piazza, "Ultra-Low-Power and High Sensitivity Resonant Micromechanical Receiver," in IEEE Sensors, Glasgow, UK, 2017.

[3] F. Pop, A. Kochhar, G. Vidal-Álvarez, and G. Piazza, "Laterally vibrating lithium niobate MEMS resonators with $30 \%$ electromechanical coupling coefficient," in IEEE Int. Conf. on Microelectromechanical Systems, Las Vegas, NV, 2017, pp. 966-969.

[4] C.T.-C. Nguyen, "Transceiver front-end architectures using vibrating micromechanical signal processors," in IEEE Topical Meeting on Silicon Monolithic Integrated Circuits in RF Systems, Ann Arbor, MI, 2001.

[5] G.K. Fedder, "CMOS-MEMS resonant mixer-filters," in IEEE Int. Electron Devices Meeting, Washington, DC, 2005.

[6] F. Chen et al., "CMOS-MEMS resonant RF mixer-filters," in IEEE Int. Conf. on Microelectromechanical Systems, Miami, FL, 2005, pp. 24-27.

[7] M. Koskenvuori, I. Tittonen, and A. Alastalo, "GHz-range FSK-reception with microelectromechanical resonators," Sensors and Actuators A, vol. 147, no. 1, Sept. 2007, pp. 346351.

[8] M. Koskenvuori and I. Tittonen, "Improvement of the conversion performance of a resonating multimode microelectromechanical mixer-filter through parametric amplification," Electron Device Lett., vol. 28, no. 11, Nov. 2007, pp. 970-972.

[9] M. J. Thompson and D. A. Horsley, "Parametrically amplified Z-axis Lorentz forced magnetometer," J. Microelectromech. Sys., vol. 20, no. 3, June 2011, pp. 702-710.

[10] R.N. Candler, M.A. Hopcroft, B. Kim, W.-T. Park, R. Melamud, M. Agarwal, G. Yama, A. Partridge, M. Lutz, and T.W. Kenny, "Long-term and accelerated life testing of a novel single-wafer vacuum encapsulation for MEMS resonators," $J$. Microelectromech. Sys., vol. 15, no. 6, 2006, pp. 1446-1456.

[11] D. Rugar and P. Grutter, "Mechanical parametric amplification and thermomechanical noise squeezing," Phys. Rev. Lett., vol. 67, no. 6, pp. 699-702, Aug. 1991.

[12] D. Shin, "Invitation to Design in the Next Epi-Seal Run: Hot Dog XV and Dorayaki V,” Stanford University, 2016.

\section{CONTACT}

*M.E. Galanko, tel: +1-412-216-5759; galanko@cmu.edu 